\title{
BAIXO NÍVEL DE ANSIEDADE DOS PACIENTES ATENDIDOS NO CURSO DE ODONTOLOGIA DE UMA INSTITUIÇÃO DE ENSINO SUPERIOR
}

\section{LOW LEVEL OF ANXIETY OF PATIENTS TREATED IN A DENTISTRY COURSE OF A HIGHER EDUCATION INSTITUTION}

\author{
Luciano Teles* \\ Dionísia Cataldo* \\ Luis Felipe Jochims Schneider*** \\ Mayra Cardoso ${ }^{\ldots+*}$ \\ Patricia Nivoloni Tannure ${ }^{* * * *}$
}

\begin{abstract}
RESUMO
Apesar de benéfico, o tratamento odontológico pode gerar ansiedade para o paciente e para o cirurgião-dentista. Se verificada além de um limite aceitável, a ansiedade pode impedir que o paciente se submeta ao tratamento, comprometendo sua qualidade de vida. Diante da relevância do assunto, objetivou-se avaliar o perfil dos pacientes atendidos nas clínicas odontológicas de uma instituição de ensino e seu nível de ansiedade diante do tratamento odontológico. Foram entrevistados 133 pacientes em tratamento nas clínicas integradas e do mestrado da Universidade Veiga de Almeida, entre março e outubro de 2015. Os pacientes responderam a um questionário com dados socioeconômicos e questões específicas para a identificação do grau de ansiedade ao tratamento odontológico, utilizando a escala DAS (Dental Anxiety Scale). A amostra foi composta de maneira equivalente por homens e mulheres, a maioria tinha mais que 45 anos $(51,1 \%)$, com renda de até 5 salários mínimos (66,9\%) e com grau de instrução superior (56,4\%). Embora 88,7\% tenham relatado algum nível de ansiedade, este foi considerado baixo. Não houve diferença estatística entre o grau de ansiedade de homens e mulheres $(p=0,458)$. Não se observou associação entre idade, gênero, renda, grau de instrução, frequência de ida ao dentista e o nível de ansiedade $(p>0,05)$. Houve uma associação significativa $(p=0,015)$ entre procedimentos que incomodam durante a consulta, como a alta rotação e a anestesia, e os níveis de ansiedade observados. Pode-se concluir que o nível de ansiedade dos pacientes atendidos foi considerado baixo; entretanto mostrou uma associação positiva com procedimentos como a alta rotação e a anestesia.
\end{abstract}

DESCRITORES: Ansiedade ao tratamento odontológico • Assistência odontológica • Estresse psicológico

\section{ABSTRACT}

Despite the fact that it is beneficial, dental treatment can cause anxiety for the patient and the dentist. If verified beyond an acceptable limit, anxiety can prevent the patient to undergo treatment, compromising his quality of life. The aim of this study was to evaluate the profile of patients treated in dental clinics of a teaching institution and their integrated and master's degree clinics from University Veiga de Almeida were interviewed between March and October 2015. The patients answered a questionnaire with socio-economic data and questions to identify the degree of anxiety to dental treatment, using the scale DAS (Dental Anxiety Scale). The sample included male $(50.4 \%)$ aged more than 45 years $(51.1 \%)$, with income of up to 5 minimum wages $(66.9 \%)$ and higher level of education (56.4\%). Although $88.7 \%$ have reported some level of anxiety, this was considered low. There was no statistical difference between the degree of anxiety of men and women $(p=0.458)$. No association was found between age, gender, income, level of education, frequency of dental visits and the level of anxiety $(p>0.05)$. There was a significant association $(p=0.015)$ between procedures that bother during dental visit, such as high-speed air turbine and anesthesia, and observed anxiety levels. It can be concluded that the level of anxiety of patients was considered low; however, it showed a positive association with procedures such as high-speed air turbine and anesthesia.

DESCRIPTORS: Dental anxiety • Dental care • Stress, psychological

* Mestrando em Odontologia da Universidade Veiga de Almeida (UVA); e-mail: teles.luciano@gmail.com

** Mestranda em Odontologia da Universidade Veiga de Almeida (UVA); e-mail: dionisiacataldo@hotmail.com

*** Doutor em Materiais Dentários e professor do Mestrado Profissional em Odontologia da Universidade Veiga de Almeida (UVA); e-mail: felipefop@gmail. com

**** Doutora em Odontologia e professora do Mestrado Profissional em Odontologia da Universidade Veiga de Almeida (UVA); e-mail: mayracardoso.mc@ gmail.com

***** Doutora em Odontologia e professora do Mestrado Profissional em Odontologia da Universidade Veiga de Almeida (UVA); e-mail: pntannure@gmail.com 


\section{N T RO DUÇÃ O}

De acordo com os resultados do Projeto SB Brasil de $2010^{1}$, 23\% da população brasileira referiram ter sofrido dor de dente nos seis meses anteriores ao levantamento epidemiológico. Sabe-se que a população brasileira nessa condição necessita de tratamento odontológico. Entretanto, o próprio tratamento pode ser um fator desencadeador de ansiedade e estresse, tanto para pacientes quanto para os cirurgiões-dentistas ${ }^{2}$, impedindo sua busca pela população.

Medo e ansiedade são sentimentos comuns a pacientes que necessitam de tratamento odontológico, representando uma barreira ao atendimento adequado do profissional ${ }^{3}$. Os procedimentos cirúrgicos realizados em ambulatório, sob anestesia local, em especial as exodontias, simbolizam, para a maioria dos pacientes, situações predisponentes a desencadear a ansiedade ${ }^{4}$. Mesmo sendo vistos erroneamente como similares, medo e ansiedade possuem diferenças entre si. O medo conceitua-se como um temor a algo ou alguma coisa que é externa e que se apresenta como um perigo real, que ameaça a integridade física ou psicológica da pessoa. Também é visto como um estado emocional de alerta ante o perigo. Já a ansiedade é caracterizada como um temor, porém sem existir um objeto real ${ }^{3}$.

Sabe-se que, até certo nível, a ansiedade é considerada normal, pois prepara o organismo para os eventos futuros, sendo uma reação natural a um estímulo. Assim, o paciente apresenta uma resposta apropriada aos estímulos, variando de acordo com sua intensidade. Por outro lado, quando a ansiedade ultrapassa as reações fisiológicas do organismo, ela é chamada patológica, podendo aumentar exageradamente a frequência cardíaca e respiratória e desencadear uma emergência médica no consultório odontológico 5 .

A ansiedade perante o tratamento odontológico apresenta um impacto significativo na qualidade da saúde bucal e, consequentemente, na qualidade de vida dos pacientes, pois causa a protelação da ida ao dentista ${ }^{6}$.

Conhecer os anseios da população atendida dentro de uma instituição de ensino valoriza o cuidado com o paciente e humaniza o atendimento realizado pelos alunos e supervisionado pelos professores. Além disso, através dessa resposta pode-se observar uma possível necessidade de mudanças na formação dos alunos e no perfil de professores. Diante da relevância do assunto abordado, o objetivo deste estudo foi avaliar o perfil dos pacientes atendidos nas clínicas odontológicas de uma instituição de ensino e o nível de ansiedade destes durante o tratamento odontológico.

\section{MÉTODOS}

Este estudo obteve a aprovação do Comitê de Ética em Pesquisa da Universidade Veiga de Almeida, sob a resolução número 365/11. Foram entrevistados pacientes que procuraram atendimento odontológico nas clínicas integradas e do mestrado do Centro de Saúde Veiga de Almeida (CSVA) da Universidade Veiga de Almeida, no período de março a outubro de 2015. Foi aplicado um questionário com dados sobre o gênero, idade, renda familiar, grau de instrução, frequência de consultas ao dentista e o procedimento odontológico que causaria desconforto, previamente publicado por Chaves et al. (2006). Em seguida, utilizou-se a Escala de Ansiedade Dentária (Dental Anxiety Scale, DAS) de Corah $^{8}$ (1969) para avaliação da ansiedade. De acordo com a escala, somando-se os valores atribuídos a cada questão, o intervalo possível de pontuação poderá variar entre 4 e 20 pontos, sendo o nível de ansiedade classificado em nulo, baixo, moderado e exacerbado.

Para análise e tratamento estatístico dos dados coletados foi usado o programa SPSS - 21.0, através do método estatístico "qui-quadrado" usando-se como nível de significância o valor de $\mathrm{p}<0,05$.

\section{RESULTADOS}

A amostra final deste estudo foi composta por 133 questionários, sendo que 67 (50,4\%) foram respondidos por homens e 66, por mulheres. A média do nível de ansiedade observada foi de 7,86 pontos, classificando a população estudada com um baixo nível de ansiedade. Não houve
TELES L

CATALDO D

SCHNEIDER LFJ

CARDOSO M

TANNURE PN

BAIXO NÍVEL

DE ANSIEDADE

DOS PACIENTES

ATENDIDOS

NO CURSO DE

ODONTOLOGIA DE

UMA INSTITUIÇÃO

DE ENSINO

SUPERIOR 
TELES L

CATALDO D

SCHNEIDER LFJ

CARDOSO M

TANNURE PN

BAIXO NÍVEL

DE ANSIEDADE

DOS PACIENTES

ATENDIDOS

NO CURSO DE

ODONTOLOGIA DE

UMA INSTITUIÇÃO

DE ENSINO SUPERIOR

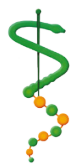

REV, ODONTOL.

UNIV, CID. SÃO PAULO

$2016 ; 28(1): 24-$

9 , JAN-ABR

Tabela 1. Caracterização dos pacientes $(N=133)$ quanto ao gênero, idade, renda familiar, grau de instrução, frequência de consultas ao dentista e procedimento odontológico que cause desconforto da amostra estudada.

\begin{tabular}{lr} 
Gênero (\%) & $\mathrm{N}(\%)$ \\
\hline Feminino & $66(49,6)$ \\
Masculino & $67(50,4)$ \\
\hline Faixa etária em anos (\%) & $65(48,9)$ \\
\hline $15-44$ & $68(51,1)$ \\
$45-65$ ou + & \\
Renda familiar mensal (\%) & $89(66,9)$ \\
Até 5 salários-mínimos & $44(33,1)$ \\
Mais que 5 salários-mínimos & \\
Grau de instrução (\%) & $25(18,8)$ \\
Analfabeto a fundamental completo & $33(24,8)$ \\
Médio incompleto a médio completo & $75(56,4)$ \\
Superior incompleto a superior completo & \\
Com que frequência você vai ao dentista? & $69(51,9)$ \\
A cada 6 meses & $5(3,8)$ \\
Não me lembro & $30(22,6)$ \\
1 vez ao ano & $21(15,8)$ \\
Somente quanto tenho dor & $8(6,0)$ \\
1 vez a cada 2 anos & \\
Dentre os procedimentos odontológicos, qual mais lhe incomoda? & $39(29,3)$ \\
Alta rotação & $37(27,8)$ \\
Anestesia & $11(8,3)$ \\
Cirurgia & $45(33,8)$ \\
Nenhum & $1(0,8)$ \\
Outro &
\end{tabular}

diferença estatística entre o grau de ansiedade de homens e mulheres $(p=0,458)$. A maioria dos pacientes (51,9\%) procurou o dentista a cada 6 meses. Dentre os procedimentos que causariam incômodo foram relatadas a alta rotação $(29,3 \%)$ e a anestesia $(27,8 \%)$. Os resultados sobre perfil socioeconômico e acerca da ansiedade de cada paciente diante dos procedimentos e situações que pudessem gerar esse sentimento encontram-se na Tabela 1. Não houve associação entre idade, gênero, renda, grau de instrução, frequência de ida ao dentista e o nível de ansiedade dos pacientes avaliados ( $p>0,05)$. Quando questionados sobre os procedimentos que incomodavam durante a consulta odontológica, observou-se uma associação significativa entre esses procedimentos e os níveis de ansiedade observados, com um p-valor de 0,015. A Tabela 2 mostra os resultados obtidos por meio da Escala de Ansiedade Dentária (DAS).

\section{I SCUSSÃO}

Os resultados deste estudo mostraram, de maneira positiva, que a população ava- liada apresentou um nível de ansiedade baixo em relação ao tratamento odontológico. Vale ressaltar ainda que este estudo teve como amostra uma população atendida por estudantes de graduação do curso de odontologia e por pós-graduandos do curso de mestrado da mesma instituição, fator que também poderia ser um causador de ansiedade aos pacientes. Por outro lado, vale ressaltar que a excelente estrutura oferecida pela instituição para o treinamento dos alunos favorece o atendimento que ocorre em horários predefinidos e com constante supervisão de um professor. Esse resultado positivo favorece o desenvolvimento dos alunos na clínica, uma vez que o atendimento se torna mais tranquilo para ambos e provavelmente proporciona mais chances de sucesso.

Vale ressaltar que não houve diferenças significativas em relação aos níveis de ansiedade entre homens e mulheres no presente estudo. Tal fato contradiz o que foi relatado por Chaves et al.7 (2006) e Bottan et al. ${ }^{9}$ (2015). Já Maniglia-Ferreira et al. ${ }^{10}$ (2004) também não destacaram diferença entre os sexos no tocante à ansiedade no tratamento odontológico. 
Tabela 2. Resultados obtidos através da Escala de Ansiedade Dentária (DAS) traduzida por Pereira et al. (2000) na população estudada ( $N=133)$.

1.Se você tiver que ir ao dentista amanhã, como você se sentiria?

a) Eu estaria antecipando uma experiência razoavelmente agradável

$32(24,1)$

b) Eu não me importaria

c) Eu me sentiria ligeiramente desconfortável

$73(54,9)$

$18(13,5)$

d) Eu temo que eu me sentiria desconfortável e teria dor

e) Eu estaria com muito medo com o que o dentista me fizesse

2.Quando você está esperando na sala de espera do dentista, como você se sente?
a) relaxado
b) meio desconfortável
c) tenso
d) ansioso
e) tão ansioso que começo a suar ou começo a me sentir mal

$76(57,1)$

$24(18,0)$

$23(17,3)$

$3(2,3)$

3.Quando você está na cadeira odontológica esperando que o dentista comece a trabalhar nos seus dentes com a turbina, como você se sente?
a) relaxado
b) meio desconfortável
c) tenso
d) ansioso
e) tão ansioso que começo a suar ou começo a me sentir mal

$64(48,1)$

$28(21,1)$

$25(18,8)$

$16(12,0)$

$0(0,0)$

4.Você está na cadeira odontológica para ter seus dentes limpos. Enquanto você aguarda o dentista pegar os instrumentos que ele usará para raspar seus dentes perto da gengiva, como você se sente?
a) relaxado
$70(52,6)$
b) meio desconfortável
$28(21,1)$
c) tenso
$20(15,0)$
d) ansioso
$13(9,8)$
e) tão ansioso que começo a suar ou começo a me sentir mal
$2(1,5)$

Em relação à renda mensal, a maioria dos pacientes $(66,9 \%)$ recebia até 5 salários mínimos. Torna-se relevante comentar que a instituição de ensino aqui reportada não é uma instituição pública ou filantrópica. Os tratamentos executados têm um custo e por esse motivo pode-se observar uma população atendida com uma condição socioeconômica de média a elevada quando comparada à média nacional. Carvalho et al. ${ }^{11}$ (2012) demonstraram que um maior nível de ansiedade está presente entre pacientes de baixa renda familiar. Assim também afirmaram Hittner e Hemmo ${ }^{12}$ (2009). Este estudo mostrou que a maioria de homens e mulheres, $60 \%$ e $48 \%$, respectivamente, afirmaram frequentar o consultório odontológico a cada seis meses. Já o estudo de Kanegane et al. ${ }^{13}$ (2006) evidenciou uma procura pelo dentista apenas quando necessário para $57,6 \%$ dos pacientes entrevistados.

Sabe-se que o nível de escolaridade está associado à renda mensal do trabalhador. Pode-se observar neste estudo que a maioria dos pacientes possuía curso superior incompleto ou completo, demonstrando uma população com um elevado nível de instrução. Provavelmente, o baixo nível de ansiedade observado nessa população estaria relacionado ao elevado nível de instrução e boa condição socioeconômica, apesar dos resultados estatísticos não mostrarem uma associação positiva entre essas variáveis. Vale ressaltar que a caneta de alta rotação e a anestesia parecem causar algum grau de ansiedade nos pacientes e merecem mais estudos a fim de controlar esses efeitos negativos.

Os pacientes também afirmaram se sentirem relaxados na sala de espera, sendo esta a resposta assinalada por $57,1 \%$ dos pacientes. Na instituição onde o estudo aconteceu sabe-se que a sala de espera
TELES L

CATALDO D

SCHNEIDER LFJ

CARDOSO M

TANNURE PN

BAIXO NÍVEL

DE ANSIEDADE

DOS PACIENTES

ATENDIDOS

NO CURSO DE

ODONTOLOGIA DE

UMA INSTITUIÇÃO

DE ENSINO

SUPERIOR

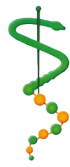

REV, ODONTOL.

UNIV. CID. SÃO

PAULO

$2016 ; 28(1): 24-$

9 , JAN-ABR 
TELES L

CATALDO D

SCHNEIDER LFJ

CARDOSO M

TANNURE PN

BAIXO NÍVEL

DE ANSIEDADE

DOS PACIENTES

ATENDIDOS

NO CURSO DE ODONTOLOGIA DE UMA INSTITUIÇÃO

DE ENSINO SUPERIOR é confortável, possui televisão, banheiros e água filtrada nos bebedouros. McGreth e Bedi ${ }^{14}$ (2004) mostraram o mesmo para $40,6 \%$ dos indivíduos investigados. O mesmo valeu para $33,33 \%$ dos pacientes analisados no estudo de Chaves et al. ${ }^{7}$ (2006). Esta pesquisa mostrou ainda que $48,1 \%$ dos pacientes afirmaram se sentirem relaxados quando já estão na cadeira do dentista, enquanto aguardam o dentista trabalhar em seus dentes com a alta rotação. Entretanto, Chaves et al.7 (2006) mostraram em sua pesquisa que $23,33 \%$ se mostraram meio desconfortáveis e 28,33\% (maior parte da amostra) se disseram tensos. Segundo Carvalho et al. ${ }^{11}$ (2012), os pacientes por eles entrevistados demonstraram que sabiam a qual procedimento seriam submetidos, sem que isso lhes causasse quaisquer transtornos de ansiedade.

O bem-estar dos pacientes e dos estudantes de graduação e pós-graduação é fundamental para um bom aprendizado. Diante da ansiedade relatada pelos pa- cientes por causa da anestesia e da alta rotação, torna-se relevante o desenvolvimento de projetos por alunos e professores com o intuito de minimizar esse desconforto. Dessa maneira, pacientes com baixo nível de ansiedade não serão impedidos de buscar atendimento odontológico a fim de alcançar uma adequada saúde bucal e geral.

\section{CONCLUSÃO}

Os pacientes atendidos nas Clínicas da UVA eram homens e mulheres em igual número, tinham mais que 45 anos $(51,1 \%)$, renda de até 5 salários mínimos $(66,9 \%)$ e com grau de instrução variando do superior incompleto ao completo (56,4\%). O nível de ansiedade dos mesmos foi considerado baixo, de acordo com a escala DAS. Foi observada uma associação positiva entre os procedimentos que incomodam durante a consulta odontológica, como a alta rotação, a anestesia e o nível de ansiedade analisada. 
TELES L

CATALDO D

SCHNEIDER LFJ

CARDOSO M

TANNURE PN

Vigilância em Saúde. Departamento de Atenção Básica. Coordenação Geral de Saúde Bucal. Projeto SB Brasil 2010: pesquisa nacional de saúde bucal: resultados principais. Brasília: Ministério da Saúde; 2011.

2. Possobon RF, Carrascoza KC, Moraes ABA, Costa Jr. AL. O tratamento odontológico como gerador de ansiedade. Psicol Estud 2007 set-dez;12(3):609-13.

3. Medeiros LA, Ramiro FMS, Lima CAA, Souza LMA, Fortes TMV, Groppo FC. Avaliação do grau de ansiedade dos pacientes antes de cirurgias orais menores. Rev odontol UNESP 2013 out.;42(5):357-63.

4. Lisboa AH, Kindl C, Pilatti GL. Nível de ansiedade em pacientes submetidos a procedimentos cirúrgicos odontológicos. Full Dent Sci 2012 3(12):400-7.

5. Pereira VZ, Barreto RC, Cavalcanti HRBB, Pereira GAS. Avaliação dos níveis de ansiedade em pacientes submetidos ao tratamento odontológico. Rev bras cien Saúde 2013 16(1):5564.

6. Kumar S, Bhargav P, Patel A, Bhati M, Balasubramanyam G, Duraiswamy $P$, et al. Does dental anxiety influence oral health-related quality of life? Observations from a cross-sectional study among adults in Udaipur district, India. J Oral Sci 2009 Jun;51(2):245-54.

7. Chaves AM, Loffredo LCM, Valsecki Júnior A, Chavez OM, Campos JIDB. Estudo epidemiológico da ansiedade dos pacientes ao tratamento odontológico Rev odontol UNESP 2006 out-dez;35(4):263-8. anxiety scale. J Dent Res 1969 JulAug;48(4):596.

8. Corah NL. Development of a dental

9. Bottan ER, Pasini B, Balestreri M, Oliveira MLRS, Marin C. Relação entre ansiedade ao tratamento odontológico e fatores sociodemográficos: estudo com adultos em Santa Catarina (Brasil). Salusvita 2015 34(1):57-70.

10. Maniglia-Ferreira C, Gurgel-Filho ED, Bönecker-Valverde G, Moura EH, Deus G, Coutinho-Filho T. Ansiedade odontológica: nível, prevalência e comportamento. RBPS 2004 17(2):515.

11. Carvalho RWF, Falcão PGCB, Campos GJL, Bastos AS, Pereira JC, Pereira MAS, et al. Ansiedade frente ao tratamento odontológico: prevalência e fatores predictores em brasileiros. Ciênc saúde coletiva 2012 jul;17(7):191522.

12. Hittner JB, Hemmo R. Psychosocial predictors of dental anxiety. J Health Psychol 2009 Jan;14(1):53-9.

13. Kanegane K, Penha SS, Borsatti MA, Rocha RG. Ansiedade ao tratamento odontológico no atendimento de rotina. RGO, Porto Alegre 2006 abrjun;54(2):111-4.

14. McGrath C, Bedi R. The association between dental anxiety and oral health-related quality of life in Britain. Community Dent Oral Epidemiol 2004 Feb;32(1):67-72.

Recebido em 02/04/2016

Aceito em 08/08/2016
BAIXO NÍVEL

DE ANSIEDADE

DOS PACIENTES

ATENDIDOS

NO CURSO DE

odONTOLOGIA DE

UMA INSTITUIÇÃO

DE ENSINO

SUPERIOR

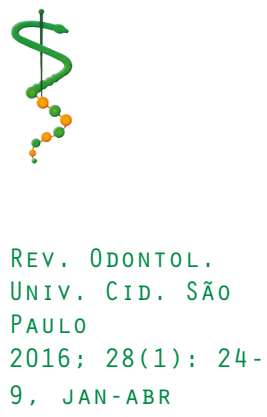

